\title{
RESULTADOS DA RECONSTRUÇÃO DO LIGAMENTO CRUZADO ANTERIOR EM ATLETAS AMADORES DE FUTEBOL
}

\author{
RESULTS OF THE ANTERIOR CRUCIATE LIGAMENT RECONSTRUCTION IN AMATEUR \\ SOCCER PLAYERS
}

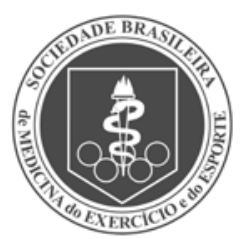

Artigo Original

\author{
RESULTADOS DE LA RECONSTRUCCIÓN DEL LIGAMENTO CRUZADO ANTERIOR EN ATLETAS \\ AMATEURS DE FÚTBOL
}

\begin{abstract}
Mauro Rodrigues dos Santos (Médico)'

Junichiro Sado Junior (Médico)² José Palmiro Neto (Médico) ${ }^{1}$ Francisco Pereira Neto (Médico) Bruno Kasuo Taia (Médico)²

1. Hospital Samaritano de Goiânia, GO, Brasil

2. Hospital dos Acidentados, Goiânia, GO, Brasil.

\section{Correspondência:}

Grupo de Joelho do Hospital dos Acidentados. Av. Paranaíba, 652, Centro, Goiânia, GO, Brasil. 74015-125.

junichirosado@uol.com.br
\end{abstract}

\section{RESUMO}

Introdução: O futebol é um esporte muito praticado em nosso meio, ocasionando uma grande incidência de lesões do ligamento cruzado anterior (LCA). Frequentemente há necessidade de tratamento cirúrgico para se permitir o retorno à sua prática, e cada vez mais são utilizados os tendões isquiotibiais como enxertos de escolha para a técnica cirúrgica. Existem poucos dados na literatura a respeito do retorno à prática do futebol em nível amador após esse tipo de tratamento. Objetivo: Avaliar o índice de retorno à prática do futebol entre atletas amadores submetidos à reconstrução do LCA, utilizando-se como enxerto os tendões semitendíneo e grácil. Métodos: Foram avaliados 97 pacientes submetidos à reconstrução do LCA com seguimento mínimo de 2 anos. A avaliação foi feita através da escala de Lysholm e do questionamento direto a respeito do retorno à prática de esportes, com ênfase ao retorno à prática do futebol amador. Resultados: 60,8\% dos pacientes operados voltaram a jogar futebol. Entre aqueles que não voltaram a praticar futebol, 9,3\% continuaram a prática de outros esportes, sem que este fato estivesse relacionado com a lesão do joelho e 11,4\% pararam de praticar esportes, relacionando o abandono da prática diretamente a sintomas no joelho operado. Um grande número (18,5\%) de pacientes informou que não retornou ao futebol devido a variadas causas relacionadas a fatores psicológicos tais como: perda de motivação, medo de uma nova lesão e mudança de estilo de vida. Conclusão: É alto o índice de pacientes que não retornam à prática do futebol após o tratamento cirúrgico por motivos psicológicos. Portanto, existe a necessidade de mais estudos nesse campo a fim de selecionarmos melhor os pacientes e, assim, melhorarmos as indicações cirúrgicas e, consequentemente, os resultados do tratamento da lesão do LCA.

Palavras-chave: traumatismos em atletas, traumatismos do joelho, reabilitação.

\begin{abstract}
Introduction: Soccer is frequently practiced in our country, causing a high incidence of anterior cruciate ligament (ACL) injuries. Often, the surgical treatment is required in order to enable the patient to return to the practice and the hamstring grafts are being widely used as the main choice for the surgical technique. There are very few articles in the literature concerning the return to amateur soccer practice after this kind of treatment.Objective: To evaluate the rate of return to soccer practice among amateur athletes who underwent $A C L$ reconstruction, using the semitendinosus and gracilis tendons as grafts. Methods: Ninety seven patients undergoing ACL reconstruction were evaluated with a minimum follow-up of 2 years. The evaluation was made by the Lysholm Scale and directly questioning regarding return to sports, with emphasis on the return to the practice of amateur soccer. Results: $60.8 \%$ of the operated patients returned to playing soccer. Among those who did not return to soccer practice, 9.3\% continued to practice other sports activities, however this fact was not related to the knee injury. Other $11.4 \%$ completely stopped playing sports due to symptoms directly related to the operated knee. A large number (18.5\%) of patients reported that they did not return to soccer practice due to many other causes related to psychological factors such as loss of motivation, fear of a re-injury, and change of lifestyle. Conclusion: The rate of patients who do not return to soccer practice after surgical treatment for psychological reasons is high. Therefore, there is a need for further studies in this field in order to best select patients and thus improve surgical indications and consequently outcomes of the treatment of $A C L$ injury.
\end{abstract}

Keywords: sports injuries, knee injuries, rehabilitation.

\section{RESUMEN}

Introducción: El fútbol amateur es uno de los deportes más practicados en nuestro medio, ocasionando una gran incidencia de lesiones del ligamento cruzado anterior (LCA). En la mayoría de esos casos hay necesidad de tratamiento quirúrgico para permitir el retorno a la práctica del fútbol, y cada vez más son utilizados los tendones isquiotibiales como injertos de elección para la técnica quirúrgica. Existen pocos datos en la literatura al respecto del retorno a la práctica del fútbol a nivel amateur después del tratamiento quirúrgico de esa lesión. Objetivo: Evaluar el 
indice de retorno a la práctica del fútbol entre atletas amateurs sometidos a la reconstrucción del LCA, utilizándose como injerto los tendones del semitendíneo y del grácil. Métodos: Fueron evaluados 97 pacientes del sexo masculino, sometidos a la reconstrucción del LCA, con seguimiento mínimo de 2 años. La evaluación fue realizada a través de la escala de Lysholm y del cuestionamiento directo al respecto del retorno a la práctica de deportes, con énfasis en el retorno a la práctica del fútbol amateur. Resultados: 60,8\% de los pacientes operados volvieron a jugar fútbol. Entre aquellos que no volvieron a practicar fútbol, 9,3\% continuaron la práctica de otros deportes, sin que ese hecho estuviese relacionado con la lesión de la rodilla, y 11,4\% pararon de practicar deportes, relacionando el abandono de la práctica directamente a los síntomas de la rodilla operada. Un gran número (18,5\%) de pacientes informó que no retornó al fútbol debido a variadas causas relacionadas a factores psicológicos, como la pérdida de motivación, miedo de una nueva lesión y cambio de estilo de vida. Conclusión: El índice de pacientes que no retornan a la práctica del fútbol después del tratamiento quirúrgico por razones psicológicas es alto. Por lo tanto, existe la necesidad de más estudios en este campo con el fin de seleccionar mejor los pacientes y así mejorar las indicaciones quirúrgicas y por consiguiente, los resultados del tratamiento de la lesión del LCA.

Palabras clave: traumatismos en atletas, traumatismos de la rodilla, rehabilitación.

Artigo recebido em 26/08/2013, aprovado em 16/12/2013.

\section{INTRODUÇÃO}

O futebol é um dos esportes mais praticados em todo o mundo e tem elevados índices de lesões nos membros inferiores ${ }^{1}$. Dentre as lesões de maior gravidade, o local mais acometido é o joelho ${ }^{2}$ sendo a lesão do ligamento cruzado anterior (LCA) uma das mais comuns ${ }^{3}$.

Na literatura, os dados acerca das lesões do LCA em praticantes de futebol amador, que são a maioria dos pacientes tratados em nosso meio, são escassos. Existem alguns trabalhos abordando resultados de tratamento cirúrgico em atletas profissionais ${ }^{3}$ e apenas $u^{4}{ }^{4}$ mostrando os resultados do tratamento cirúrgico em atletas amadores utilizando o terço central do tendão patelar.

O objetivo deste trabalho foi de avaliar o índice de retorno à prática de futebol em atletas amadores com diagnóstico de lesão do LCA e que foram submetidos a reconstrução artroscópica utilizando os tendões isquiotibiais (semitendíneo e grácil).

\section{MATERIAIS E MÉTODOS}

O estudo foi realizado por meio de levantamento de casos, de forma retrospectiva e foi baseado no questionário de Lysholm feito a 97 pacientes operados por dois cirurgiões membros da Sociedade Brasileira de Cirurgia do Joelho, em seus respectivos hospitais, no período de junho de 2008 a dezembro de 2009.

Foram incluídos no estudo somente atletas amadores que tiveram a lesão do LCA durante a prática do futebol e estavam impossibilitados de praticar o mesmo.

Foram excluídos do estudo atletas profissionais de qualquer modalidade, casos com lesões ligamentares múltiplas, pacientes submetidos a procedimentos cirúrgicos prévios no joelho, pacientes do sexo feminino e pacientes submetidos ao tratamento cirúrgico com outros tipos de enxerto.

Todos os pacientes tinham diagnóstico de lesão do LCA que foi constatado na anamnese e do exame físico, provas ligamentares positivas para a lesão (teste de Lachmann, gaveta anterior e pivot Shift). O diagnóstico foi confirmado por ressonância nuclear magnética (RM), exame físico sob anestesia e na inspeção artroscópica intra-operatória.

A técnica cirúrgica utilizada foi a reconstrução artroscópica do LCA utilizando-se os tendões isquiotibiais semitendinoso e gracilis, confeccionados de forma quádrupla e fixada de três formas diferentes no fêmur: placa extracortical, parafuso transverso e parafuso de interferência, todos de titânio. Na tíbia todos foram fixados com parafuso de interferência de titânio. As lesões associadas de meniscos foram documentadas e tratadas com meniscectomia parcial. As lesões condrais também foram documentadas e as mais acentuadas, grau 3 e 4 da
International Cartilage Repair Society (ICRS) tratadas com abrasão e/ou microfraturas. O processo de reabilitação em sua grande maioria foi supervisionado por equipes de fisioterapeutas treinados, porém não foi critério de inclusão ou exclusão do estudo.

Não houve nenhuma complicação grave durante o intra e o pós-operatório. Nenhum paciente foi reoperado até o período da avaliação.

A avaliação pós-operatória foi baseada no questionário de Lysholm ${ }^{5}$ e por questões que se referiam ao objetivo do estudo, ou seja, o nível de prática do futebol amador antes e após a reconstrução do LCA. O questionário foi aplicado através de contato telefônico diretamente com o paciente e foi realizado por três médicos que participaram do estudo, porém não participaram das cirurgias realizadas.

\section{Análise Estatística}

Os dados foram digitados e preparados no software Excel para Windows para posterior análise em programa estatístico Statistical Package for the Social Sciences - SPSS, versão 17.0.

Nas tabelas foram apresentadas as frequências absolutas e o percentual encontrado em cada uma das variáveis em estudo.

Foi usado o teste KS (Kolmogorov Smirnov) para testar a normalidade dos dados. Os dados não apresentaram distribuição normal.

Para verificar a existência de significância estatística foram utilizados os testes de Fisher, de Mann-Whitney, de Kruskal-Wallis e o de correlação de Pearson de acordo com o intuito de comparação.

O teste de Fisher foi usado para verificar existência de diferença significativa nos grupos quanto às variáveis: lado acometido, lesões meniscais e lesões condrais associadas, resultado do questionário de Lysholm e frequência de prática de futebol antes da lesão.

O teste Qui-quadrado foi usado para testar a diferença de pontuação de Lysholm quanto aos métodos de fixação femoral empregados.

O teste Mann-Whitney foi usado para comparar a pontuação de Lysholm entre os pacientes que retornaram e os que não retornaram ao futebol amador.

O teste Kruskal-Wallis foi usado para verificar diferença significativa ou não entre o grau de retorno ao futebol, quanto ao tempo de lesão, a idade e a pontuação de Lysholm.

\section{RESULTADOS}

Dos 97 pacientes investigados, 66 pacientes (68\%), eram praticantes de futebol amador ocasional, com uma frequência de até duas vezes por semana, outros $23(23,7 \%)$ referiram praticar a atividade mais que duas vezes por semana, porém sem a característica de competição nessas partidas e o 
restante da amostra, oito pacientes (8,3\%), afirmaram participar de atividades competitivas amadoras regulares, porém sem serem atletas profissionais.

A idade variou entre 15 e 58 anos, sendo a média de 31,9 anos com desvio padrão de \pm 9,9 anos.

O tempo transcorrido entre a lesão até a cirurgia variou entre menos de um mês, até 243,5 meses, com média de 24,9 meses e desvio padrão \pm 40,21 meses.

A incidência de lesões no joelho direito ocorreu em 65 pacientes (67\%) da amostra e do lado esquerdo em 32 pacientes (33\%) com $p=0,850$, ou seja, sem diferença estatística significativa.

Com relação às lesões associadas, 49 pacientes (50,5\%) apresentaram lesão do menisco medial e 23 pacientes ( $23,7 \%$ ) do menisco lateral. 16 pacientes (16,5\%) apresentaram lesão condral medial ou lateral e seis pacientes $(6,2 \%)$ apresentaram lesão condral da tróclea ou da patela.

Na análise do objetivo principal deste estudo, 70,1\% dos pacientes voltaram a praticar alguma atividade física, porém apenas 60,8\% voltaram a jogar futebol, havendo variação entre o retorno no mesmo nível ou intensidade, abaixo ou acima do nível pré-lesão (tabela 1). 0 maior índice de desistência da prática esportiva ocorreu naquele grupo de pessoas que já praticavam a atividade apenas esporadicamente. Apesar dessa observação, a amostra não foi estatisticamente suficiente para compararmos o grau de retorno ao futebol entre os três grupos separados de acordo com a frequência da prática do futebol.

Entre os 38 pacientes (39,2\%) que não voltaram a jogar futebol, observamos que nove pacientes $(9,3 \%)$ continuaram com a prática de outros esportes, sem este fato estar relacionado à lesão do joelho, 18 pacientes (18,5\%) informaram que não retornaram à prática de esportes devido a variadas causas tais como falta de tempo, medo de nova lesão, o avançar da idade entre outros, e apenas 11 pacientes $(11,4 \%)$ relacionaram o abandono da prática diretamente ao joelho operado, relatando dor, insegurança e/ou falseio.

A idade do paciente e o tempo de evolução da lesão não foram fatores determinante estatisticamente em nossa amostra para o retorno às atividades futebolística, com $p=0,056$ (tabelas 2 e 3).

Tabela 1. Distribuição dos pacientes em relação à frequência da prática do futebol antes da lesão e o índice de retorno ao futebol amador após a cirurgia.

\begin{tabular}{c|c|c|c|c}
\hline \multirow{2}{*}{$\begin{array}{c}\text { Retorno ao futebol } \\
\text { amador }\end{array}$} & \multicolumn{3}{|c|}{ Futebol antes da lesão } & \multirow{2}{*}{ Total } \\
\cline { 2 - 4 } & $\begin{array}{c}\text { Comp. } \\
\text { amadoras }\end{array}$ & $\begin{array}{c}\text { Máximo } \\
\text { 2x semana }\end{array}$ & $\begin{array}{c}\text { Mais que } \\
\text { 2x semana }\end{array}$ & \\
\hline Não retornou & $2 / 25,0 \%$ & $10 / 43,5 \%$ & $26 / 39,2 \%$ & $38 / 39,2 \%$ \\
\hline Competições amadoras & $4 / 50,0 \%$ & $-/ 0,0 \%$ & $-/ 0,0 \%$ & $4 / 4,1 \%$ \\
\hline Máximo 2x semana & $1 / 12,5 \%$ & $5 / 21,7 \%$ & $1 / 1,5 \%$ & $7 / 7,2 \%$ \\
\hline Mais que 2x semana & $1 / 12,5 \%$ & $8 / 34,8 \%$ & $39 / 59,1 \%$ & $48 / 49,5 \%$ \\
\hline Total & $8 / 100,0 \%$ & $23 / 100,0 \%$ & $66 / 100,0 \%$ & $97 / 100,0 \%$ \\
\hline
\end{tabular}

Tabela 2. Média de idade quanto ao grau de retorno ao esporte.

\begin{tabular}{c|c|c|c}
\hline \multirow{2}{*}{$\begin{array}{c}\text { Retorno ao futebol } \\
\text { amador }\end{array}$} & \multicolumn{2}{|c|}{ Idade } & \multirow{2}{*}{$\mathbf{p}$} \\
\cline { 2 - 3 } & Média \pm DP & Mediana (IC95\%) & \\
\hline Não retornou & $32,13 \pm 11,07$ & $28,5(28,5-35,8)$ & \\
\cline { 1 - 3 } Competições amadoras & $31,00 \pm 10,10$ & $31,5(14,9-47,1)$ & \multirow{2}{*}{0,056} \\
\cline { 1 - 3 } Máximo 2x semana & $24,57 \pm 5,25$ & $24,0(19,7-29,4)$ & \\
\hline Mais que 2x semana & $33,00 \pm 9,28$ & $33,0(30,3-35,7)$ & \\
\hline Teste: Kruskall-Wallis (obs:no teste não estão incluídos os que não retornaram ao futebol).
\end{tabular}

Tabela 3. Média do tempo de lesão quanto ao grau de retorno ao esporte.

\begin{tabular}{c|c|c|c}
\hline \multirow{2}{*}{$\begin{array}{c}\text { Retorno ao futebol } \\
\text { amador }\end{array}$} & \multicolumn{2}{|c|}{ Tempo de lesão } & \multirow{2}{*}{$\mathbf{p}$} \\
\cline { 2 - 3 } & Média \pm DP & Mediana(IC95\%) & \\
\hline Não retornou & $26,12 \pm 39,92$ & $5,0(13,0-39,2)$ & \\
\hline Competições amadoras & $44,14 \pm 71,39$ & $9,6(-69,4-157,7)$ & \multirow{2}{*}{0,550} \\
\hline Máximo 2x semana & $14,07 \pm 18,59$ & $6,1(-3,1-31,3)$ & \\
\hline Mais que 2x semana & $23,90 \pm 40,34$ & $8,1(12,2-35,6)$ & \\
\hline
\end{tabular}

O teste de Lysholm teve correlação estatisticamente significativa com o retorno à prática de futebol. No grupo de pacientes que conseguiu retornar à prática desse esporte, a pontuação do questionário de Lysholm foi significativamente maior do que no grupo de pacientes que não retornaram (tabela 4).

Por fim separamos os pacientes operados em três grupos de acordo com o método de fixação do enxerto no fêmur para avaliarmos se houve alguma diferença de resultados entre esses grupos.

O método de fixação femoral empregado foi a placa extracortical em 38 pacientes, parafuso transverso em 48 e parafuso de interferência em 11 pacientes.

Pelo teste qui-quadrado observamos que não existe diferença significativa na pontuação de Lysholm, entre os métodos de fixação femoral empregados. Para tal conclusão foram testadas as pontuações com resultados excelentes e bom, uma vez que foram insuficientes para testes os resultados regulares e ruins.

Os métodos de fixação femoral empregados não apresentaram diferença significativa na pontuação final do questionário, com $\mathrm{p}=0,263$ (tabela 5).

Já na avaliação do retorno ao futebol entre os métodos de fixação utilizamos o teste de Fisher, não sendo possível incluir nesta comparação os pacientes operados com parafuso de interferência no fêmur devido ao reduzido número da amostra. Observamos que o grupo do parafuso transverso obteve resultados estatisticamente significativos melhores do que o grupo da placa extracortical, com índice de retorno de $68,7 \%$, contra $50 \%$ do outro grupo $(p=0,038)$.

\begin{tabular}{c|c|c|c} 
Tabela 4. Média da pontuação de Lysholm quanto ao grau de retorno ao esporte. \\
\cline { 1 - 3 } $\begin{array}{c}\text { Retorno ao futebol } \\
\text { amador }\end{array}$ & \multicolumn{2}{|c|}{ Pontuação de Lysholm } & \multirow{2}{*}{ p } \\
\cline { 2 - 3 } & Média \pm DP & Mediana(IC95\%) & \\
\hline Não retornou & $92,84 \pm 10,22$ & $95,0(89,5-96,2)$ & \multirow{2}{*}{$0,003^{*}$} \\
\hline Retornou ao Futebol & $97,61 \pm 4,36$ & $100,0(96,5-98,7)$ & \\
\hline
\end{tabular}

Teste: Man-whitney.

Tabela 5. Resultado do questionário de Lysholm de acordo com o método de fixação femoral do enxerto.

\begin{tabular}{c|c|c|c}
\hline $\begin{array}{c}\text { Resultado do } \\
\text { questionário }\end{array}$ & $\begin{array}{c}\text { Placa extracortical } \\
(\mathbf{n}=\mathbf{3 8})\end{array}$ & $\begin{array}{c}\text { Paraf. Transverso } \\
(\mathbf{n}=\mathbf{4 8})\end{array}$ & $\begin{array}{c}\text { Paraf. de } \\
\text { Interferência }(\mathbf{n}=\mathbf{1 1})\end{array}$ \\
\hline Excelente & $26 / 68,4 \%$ & $40 / 83,3 \%$ & $10 / 90,9 \%$ \\
\hline Bom & $9 / 23,7 \%$ & $6 / 12,5 \%$ & $-/ 0,0 \%$ \\
\hline Regular & $3 / 7,9 \%$ & $2 / 4,2 \%$ & $-/ 0,0 \%$ \\
\hline Ruim & $-/ 0,0 \%$ & $-/ 0,0 \%$ & $1 / 9,1 \%$ \\
\hline
\end{tabular}

Teste usado: Qui-Quadrado. $P=0,263$.

\section{DISCUSSÃO}

A incidência de lesões ortopédicas em atletas de futebol em todo o mundo já foi estudado por muitos pesquisadores ${ }^{1-3}$. Também há alguns estudos que avaliam o retorno ao esporte profissional, incluindo o futebol, após o tratamento cirúrgico da lesão do LCA ${ }^{3,6-9}$. Porém encontramos apenas um estudo da incidência de retorno especificamente dos pacientes que praticam futebol amador, após reconstrução cirúrgica do LCA com terço central do tendão patelar ${ }^{4}$. Não encontramos nenhum estudo avaliando o índice de retorno à prática de futebol em atleta amadores utilizando os tendões isquiotibiais. O crescente uso dessa técnica por parte dos cirurgiões no mundo todo e no Brasil nos levou a fazer este levantamento.

A lesão do LCA ocorre na maioria dos casos por trauma indireto (entorse), sem contato físico direito. Portanto, sua maior incidência se dá nos esportes em que há grande quantidade de movimentos rotacionais tais como o futebol, basquetebol e o esqui alpino ${ }^{11}$. Ocasionalmente também pode ocorrer por um mecanismo de estresse em varo com rotação interna, assim como em movimentos de hiperextensão e outros traumas diretos. 
O potencial de reparo espontâneo após a lesão do LCA é muito limitado. Apesar disso, alguns autores ${ }^{13,15}$ mostram que o tratamento conservador pode ter bons resultados funcionais em pacientes cuidadosamente selecionados e reabilitados com programas específicos de fisioterapia. No entanto, a maioria dos pacientes que desejam retornar às atividades físicas que envolvem movimentos múltiplos rotacionais, saltos e mudança brusca de direção como é o caso do futebol, necessitam de tratamento cirúrgico a fim de restabelecer a prática desse esporte $^{12}$, pois nesses casos, quando a estabilidade articular não é restabelecida, podem ocorrer lesões meniscais e condrais de forma progressiva levando a uma má evolução a longo prazo ${ }^{10}$.

Em nossa casuística, a idade média dos pacientes operados foi de 31,9 anos, semelhante à casuística de $\operatorname{Ardern}^{18} \mathrm{com}$ média de 32,5 anos, porém bem acima da idade média de outras séries envolvendo atletas de nível competitivo nas quais a idade média variou de 24,33,19 a 28 anos de idad ${ }^{8,20}$. Apesar de termos excluído as pacientes do sexo feminino do estudo devido ao pouco número de casos, os trabalhos epidemiológicos que comparam o risco de lesão do LCA de acordo com o gênero, mostram taxas de incidência que variam de 2,5 até 4,2 vezes maior em mulheres em comparação com os homens, em esportes como basquetebol, handebol e futebol 3,11

As causas exatas para essa maior incidência ainda são motivo de controvérsia e objeto de muitos estudos, mas já existem programas de treinamento físico específicos que mostram resultados promissores na prevenção dessa lesão nesse grupo específico de atletas ${ }^{21,22}$ e em praticantes de futebol de maneira geral ${ }^{14}$.

A incidência de lesão meniscal associada à lesão do LCA apresenta grande variação na literatura, entre 16\% e 82\% sendo que nos casos agudos, o menisco lateral apresenta uma incidência maior de lesão e nos casos crônicos a lesão do menisco medial torna-se mais frequen$\mathrm{te}^{3,23,24}$. Esses dados coincidem com nossos achados nos quais houve mais que o dobro de lesão no menisco medial (50,5\%) em comparação com a presença de lesão do menisco lateral $(23,7 \%)$, uma vez que nossa amostra apresentou uma média de 24,9 meses transcorridos entre a lesão ligamentar e o tratamento cirúrgico, ou seja, eram na maioria casos crônicos.

Os enxertos que podem ser utilizados na reconstrução do LCA são muitos, sendo os mais comuns o terço central do tendão patelar e os tendões flexores semitendíneo e grácil. A evolução dos métodos de fixação dos tendões isquiotibiais tem aumentado muito a utilização desse enxerto nos últimos anos, inclusive entre atletas profissionais ${ }^{19}$.

Walden et al. ${ }^{3}$ em recente levantamento epidemiológico entre jogadores de futebol da primeira divisão da Europa, constatou a utilização de tendões flexores em 67\% dos atletas profissionais operados na Suécia. A maioria dos trabalhos comparativos entre as duas técnicas demonstram resultados funcionais iguais, independentemente do perfil dos pacientes em relação ao sexo e ao tipo de prática esportiva 3,17,20.

Bons resultados de retorno ao esporte também podem ser conseguidos utilizando-se outros enxertos tal como o tendão quadriciptal ${ }^{16}$. Cada técnica apresenta vantagens e desvantagens, de forma que a escolha sobre qual técnica utilizar baseia-se em fatores específicos de cada paciente e na escolha pessoal do cirurgião.

A escolha do método de fixação também parece não influenciar nos resultados finais do tratamento ${ }^{12,26-28}$. Price et al., em trabalho prospectivo e randomizado ${ }^{28}$ não observaram diferença estatisticamente significante entre o uso de parafuso transverso no fêmur ou de Endobutton ${ }^{\circledR}$ Com seguimento de 2 anos e utilizando o escore IKDC e artrômetro KT-1000 ${ }^{\text {TM }}$ (MEDmetric - San Diego , CA, USA).

Yosmaoglua et al., também fizeram estudo comparativo entre as duas técnicas ${ }^{27}$ e não encontraram diferenças no resultado final. Em nosso trabalho, ao contrário da literatura pesquisada, observamos diferença significativa entre os métodos de fixação, sendo os melhores resultados obtidos pelos pacientes com fixação por parafuso transverso obtendo um índice de retorno de 68,7\%. Porém, não podemos concluir que há superioridade de um método em relação ao outro pelo fato do nosso trabalho ser retrospectivo, com número de pacientes reduzido e com método de avaliação subjetivo.

O teste de Lysholm teve correlação estatisticamente significativa com o retorno à prática de futebol, pois no grupo de pacientes que conseguiu retornar à prática desse esporte, a pontuação foi significativamente maior do que no grupo de pacientes que não retornaram, mostrando que apesar de ser subjetivo, apresentou grande correlação com o resultado funcional dos pacientes. Para avaliarmos especificamente o retorno ao futebol, dividimos os pacientes em três grupos de acordo com a frequência da prática do esporte.

Existem escores específicos de avaliação de prática de atividade física tais como o Tegner, porém o mesmo não foi utilizado, pois nossa amostra envolveu especificamente pacientes praticantes de futebol amador. Portanto, não encontramos nenhum questionário que atendesse nossos objetivos.

Alguns trabalhos na literatura avaliaram o índice de retorno a diversos esportes em nível competitivo e/ou profissional após a reconstrução do LCA. No trabalho de Arden et al. ${ }^{8}$, 64\% dos 503 atletas (sendo $86 \%$ desses de nível competitivo) que se submeteram a reconstrução do LCA conseguiram retornar à prática de diversos esportes (basquete, netball, futebol) porém apenas 33,4\% retornaram ao nível competitivo. O mesmo autor encontrou resultados semelhantes em outro trabaIho ${ }^{18}$. Busfield et al. ${ }^{6}$ verificaram taxa de retorno de $78 \%$ entre atletas pesquisados na National Basketball Association (NBA), liga principal de basquete profissional americana. A mesma taxa de $78 \%$ de retorno ao esporte entre 18 atletas profissionais foi observada na liga feminina de basquete dos Estados Unidos, a WNBA, pelos autores Nandari et al.?.

Gobbi et al. ${ }^{9}$ encontraram taxas de retorno de $65 \%$ ao mesmo nível de prática de diversos esportes entre 80 atletas de nível competitivo submetidos a reconstrução do LCA com enxerto semitendíneo quádruplo. No trabalho de Nakayama et al. ${ }^{19} 92 \%$ entre 50 atletas de nível competitivo de diversos esportes retornaram à prática no mesmo nível anterior à lesão.

Outros trabalhos avaliaram apenas atletas de nível amador submetidos a reconstrução do LCA. Aglietti et al. ${ }^{17}$ encontraram taxas de 72\% de retorno à prática de vários esportes entre atletas amadores operados. Gobbi et al. ${ }^{20}$, encontraram taxa de retorno de 65\%. Em nosso meio, Hernandez et al. ${ }^{12}$, encontraram taxas de retorno ao esporte semelhantes ao nível pré-lesão em pacientes submetidos a reconstrução do LCA com terço central do tendão patelar. Em nossa casuística 70,1\% dos pacientes operados voltaram a praticar algum esporte.

Na análise de trabalhos que avaliaram apenas atletas de futebol, encontramos resultados como os de Walden et al. ${ }^{3}$ que encontraram índices de retorno de $89 \%$ entre 78 jogadores profissionais de futebol de clubes da primeira divisão da Europa submetidos a reconstrução do LCA, apesar de terem considerado como retorno a realização de uma única partida de futebol. No trabalho de Paz envolvendo 82 jogadores recreativos de futebol submetidos a reconstrução do LCA com terço central do tendão patelar, $88 \%$ retornaram à prática do futebol. Nossa amostra apresentou índice de retorno global à prática do futebol amador de 60,8 \%.

Em recente metanálise envolvendo 48 estudos com 5770 participantes, Ardern et al.29 mostraram que o índice de retorno á prática es- 
portiva após reconstrução do LCA é em torno de 82\% porém somente $63 \%$ ao mesmo nível pré-lesão. Nos esportistas de nível competitivo apenas 44\% retornaram ao mesmo nível de prática.

A idade do paciente e o tempo de lesão (aguda ou crônica) não foi fator determinante estatisticamente em nossa amostra para o retorno às atividades futebolísticas. Já no trabalho de Ardern et al. ${ }^{18}$, apesar da média de idade ser semelhante à dos nossos pacientes, foram encontradas taxas de retorno ao esporte (diversas modalidades esportivas) maiores nos pacientes mais jovens.

Entre os 38 pacientes (39,2\% do total) que não voltaram a jogar futebol, observamos que nove pacientes (9,3\% do total) continuaram com a prática de outros esportes, sem este fato estar relacionado à lesão do joelho. Portanto, se somarmos estes aos que efetivamente voltaram a jogar futebol, temos 68 pacientes $(70,1 \%$ do total) que voltaram à prática de qualquer tipo de esporte. Apenas 11 pacientes $(11,4 \%$ do total) relacionaram a abandono da prática diretamente ao joelho operado, relatando dor, insegurança e/ou falseio residual. Os outros 18 pacientes (18,5\% do total) informaram que não retornaram ao futebol devido a variadas causas não relacionadas ao joelho tais como a falta de tempo, o avançar da idade, o medo de nova lesão, entre outros. Dentro deste último grupo se encontram aqueles pacientes envolvidos por fatores psicológicos para a interrupção da prática do esporte, que já foram estudados por diversos autores ${ }^{3,30}$.Gobbi et al. ${ }^{9}$ também encontraram $35 \%$ de pacientes que não retornaram ao mesmo nível de prática de esportes devido a mudanças no estilo de vida ou medo de uma nova lesão. Em outro trabalho ${ }^{20}$ o mesmo autor constatou, através do uso de um questionário de avaliação psicológica, que há correlação direta entre o grau de disposição e compromisso do paciente e o retorno à prática do esporte. Nossos resultados também são compatíveis com a metanálise de Ardern et al. ${ }^{29}$ que encontraram como causa principal para o abandono dos

\section{REFERÊNCIAS}

1. Inklaar H. Soccer injuries : Incidence and severity. Sports Med. 1994;18:55-73.

2. Chomiak J, Junge A, Peterson L,Dvorak J. Severe injuries in football players influencing factors. Am J Sports Med. 2000;28:58-68.

3. Waldén $M, H a ̈ g g l u n d ~ M$, Magnusson $H$, Ekstrand J. Anterior cruciate ligament injury in elite football: a prospective three-cohort study. Knee Surg Sports Traumatol Arthrosc. 2011;19(1):11-9

4. Paz MC. Reconstrucción artroscópica del LCA en jugadores de fútbol. Resultados con seguimiento entre 2 y 9 años. Rev Argent Artroscop 1996;3(7):406-11.

5. Lysholm J, Gillquist J. Evaluation of knee ligament surgery results with a special emphasis on use of a scoring scale. Am J Sports Med.1982;10(3):150-4.

6. Busfield BT, Kharrazi FD, Starkey C, Lombardo SJ, Seegmiller J.

7. Performance outcomes of anterior cruciate ligament reconstruction in the National Basketball Association. Arthroscopy. 2009;25(8):825-30

8. Namdari S, Scott K Ba, Milby A, Baldwin K, Lee GC. Athletic performance after ACL reconstruction in the Women's National Basketball Association. Phys Sportsmed. 2011;39(1):36-41

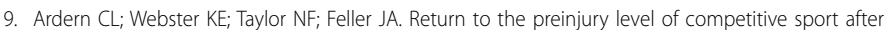
anterior cruciate ligament reconstruction surgery: two-thirds of patients have not returned by 12 months after surgery. Am J Sports Med. 2011; 39(3):538-43r.

10. Gobbi A, Tuy B, Mahajan S, Panuncialman I, Quadrupled Bone-Semitendinosus Anterior Cruciate Ligament Reconstruction: A Clinical Investigation in a Group of Athletes. Arthroscopy. 2003;7:691-9.

11. Amatuzzi MM, Camanho GL. Evoluçäo das lesöes isoladas do ligamento cruzado anterior. Rev. Bras. Ortop. 1988;23(7):183-6.

12. Agel J, Arendt EA, Bershadsky B. Anterior cruciate ligament injury in national collegiate athletic association basketball and soccer. Am J Sports Med. 2005;33:524-31

13. Hernandez AJ, Rezende MU,Góis SL; Grisende SC. Avaliaçäo funcional e do nível de atividade física nas reconstruçöes do ligamento cruzado anterior. Rev Bras Ortop; 1996;31 (12): 990-4.

14. Amatuzzi MM, Albuquerque RFM, Amatuzzi ML, Sasaki SU. O tratamento cirúrgico é imperativo na lesão do ligamento cruzado anterior? Há lugar para o tratamento conservador?.Rev Bras Ortop. 2007 ;42 (8):231-6.

15. Brito J, Soares J, Rebelo A.N. Prevenção de lesões do ligamento cruzado anterior em futebolistas. Rev Bras Med Esport. 2009;15(1):62-9.

16. Kaplan $Y$. Identifying individuals with an anterior cruciate ligament-deficient knee as copers and noncopers: a narrative literature review. J Orthop Sports Phys Ther. 2011;41(10):758-66.

17. Guimarães MV, Honório L,Terra DL. Reconstrução do ligamento cruzado anterior com terço central do tendão do músculo quadríceps: Análise de resultados após 10 anos. Rev Bras Ortop. 2009;44(4):306-12.

18. Aglietti P, Giron F, et al. Anterior Cruciate ligament reconstruction: bone-patellar tendon-bone compared esportes o medo de uma nova lesão (19\% dos pacientes que não retornaram à prática esportiva) assim como mudanças no estilo de vida (18\% dos pacientes). Outros fatores como cinesiofobia, depressão, ansiedade, distúrbio do humor, etc., também foram estudados por Brand et al. ${ }^{30}$.Os autores fazem um levantamento da literatura sobre os fatores psicológicos envolvidos no retorno ao esporte em pacientes submetidos a reconstrução do LCA e mostram a correlação direta da pontuação em questionários de avaliação de aspectos psicológicos tais como o Knee Self-Efficacy Scale (K-SES), o Tampa Scale of Kinesiophobia (TSK) no retorno ao esporte. Tais fatores devem ser cada vez mais estudados e observados pelos cirurgiões a fim de utilizarmos ferramentas que ajudem na seleção adequada dos pacientes, individualizando a indicação do tratamento (conservador ou cirúrgico) e criando terapias ou estímulos que aumentem a adesão dos pacientes a todo o processo, melhorando os resultados do tratamento da lesão do LCA.

\section{CONCLUSÕES}

Os índices de retorno à prática de futebol em atletas amadores submetidos à reconstrução do ligamento cruzado anterior no presente estudo foi de 60,8\%. Uma parte dos pacientes (18,5\%) relataram motivos não relacionados ao joelho operado para o abandono da prática do futebol, podendo esse fato estar relacionado a motivos tais como fatores psicológicos ainda pouco estudados. Existe a necessidade de mais estudos nesse campo a fim de melhorarmos a seleção dos pacientes e, consequentemente, as indicações e os resultados do tratamento da lesão do LCA.

Todos os autores declararam não haver qualquer potencial conflito de interesses referente a este artigo. with double semitendinosus and gracilis tendon grafts a prospective, randomized clinical trial. Journal of Bone and Joint Surgery 2004; 86-A(10):2143-2155.

19. Ardern C,Taylor N,Feller J,Webster K. Return-to-Sport Outcomes at 2 to 7 Years

20. After Anterior Cruciate Ligament Reconstruction Surgery. Am J Sports Med. 2012;40(1):41-8.

21. Nakayama Y; Shirai Y; Narita T; Mori A; Kobayashi K. Knee functions and a return to sports activity in competitive athletes following anterior cruciate ligament reconstruction. J Nippon Med Sch. 2000;67(3):172-6.

22. Gobbi A, Francisco R. Factors affecting return to sports after anterior cruciate ligament reconstruction with patellar tendon and hamstring graft: a prospective clinical investigation. Knee Surg Sports Traumatol Arthrosc. 2006;14(10):1021-8.

23. Mandelbaum BR, Silvers $\mathrm{HJ}$, Watanabe DS, et al. Effectiveness of a neuromuscular and proprioceptive training program in preventing anterior cruciate ligament injuries in female athletes. 2-year follow-up. Am J Sports Med. 2005;33:1003-10.

24. Myklebust $\mathrm{G}$, Engebretsen $\mathrm{L}$, Braekken $\mathbb{H}$, et al. Prevention of anterior cruciate ligament injuries in female team handball players: a prospective intervention study over three seasons. Clin J Sports Med. 2003;13:71-

25. Rocha I, Moraes T, Rezende MU ,Pécora JR. Avaliação da evolução de lesões associadas à lesão do ligamento cruzado anterior. Acta Ortop Bras. 2007;15(2):105-8.

26. Smith JP, Barrett GR. Medial and lateral meniscal tear patterns in anterior cruciate ligament-deficient knees. Am J Sports Med. 2001; 29:415-9.

27. Ejerhed L, Kartus J, Sernert N, Köhler K, Karlsson J. Patellar tendon or semitendinosus tendon autografts for anterior cruciate ligament reconstruction? A prospective randomized study with a two year followup. Am J Sports Med. 2003;31:19-25.

28. Guarilha E, Caldeira P, Neto O,Navarro M, Milani A, Carneiro M. Estudo prospectivo randomizado entre as fixações transversas e extracorticais nas reconstruções do ligamento cruzado anterior. Rev Bras Ortop. 2012;47(3):354-8.

29. Yosmaoglua HB; Baltaci G; Kaya D; Ozer H; Atay A. Comparison of functional outcomes of two anterior cruciate ligament reconstruction methods with hamstring tendon graft. Acta Orthop Traumatol Turc. 2011;45(4):240-7.

30. Price R, Stoney J, Brown G. Prospective randomized comparison of endobutton versus cross-pin femoral fixation in hamstring anterior cruciate ligament reconstruction with 2-year follow-up. ANZ J Surg. 2010;80(3):162-5.

31. Ardern CL; Webster KE; Taylor NF; Feller JA. Return to sport following anterior cruciate ligament reconstruction surgery: a systematic review and meta-analysis of the state of play. Br J Sports Med. 2011:45(7):596-606.

32. Brand E, Nyland J. Patient outcomes following anterior cruciate ligament reconstruction: The Influence of Psychological Factors. Orthopedics. 2009;32(5):335. 